This item was submitted to Loughborough's Research Repository by the author.

Items in Figshare are protected by copyright, with all rights reserved, unless otherwise indicated.

\title{
Information based mobile sensor planning for source term estimation of a non-continuous atmospheric release
}

PLEASE CITE THE PUBLISHED VERSION

https://doi.org/10.1109/ICRA.2018.8460686

PUBLISHER

IEEE

VERSION

AM (Accepted Manuscript)

LICENCE

CC BY-NC-ND 4.0

\section{REPOSITORY RECORD}

Hutchinson, Michael, Cunjia Liu, and Wen-Hua Chen. 2018. "Information Based Mobile Sensor Planning for Source Term Estimation of a Non-continuous Atmospheric Release”. figshare.

https://hdl.handle.net/2134/28377. 


\title{
Information based mobile sensor planning for source term estimation of a non-continuous atmospheric release
}

\author{
Michael Hutchinson, Cunjia Liu and Wen-Hua Chen
}

\begin{abstract}
This paper presents a method to estimate the original location and the mass of an instantaneous release of hazardous material into the atmosphere. It is formulated as an inverse problem, where concentration observations from a mobile sensor are fused with meteorological information and a Gaussian puff dispersion model to characterise the source. Bayes' theorem is used to estimate the parameters of the release taking into account the uncertainty that exists in the dispersion parameters and meteorological variables. An information based reward is used to guide an unmanned aerial vehicle equipped with a chemical sensor to the expected most informative measurement locations. Simulation results compare the performance between a single mobile sensor with various amounts of static sensors.
\end{abstract}

\section{INTRODUCTION}

Searching for the source of an atmospheric release of dispersing material is an important task for mankind and also in the natural world. The reason for finding the source may vary, for humans it is often in an emergency response to some hazardous release, searching for useful resources or inspecting an area for mines [1]. In nature the intentions are more virtuous, such as searching for a food source or even a mate [2]. In most cases, especially within the literature on source localisation, it is assumed that the source is continuously emitting. This assumption simplifies the problem, allowing techniques to attempt to track the concentration of the material to its source [3].

In this work, a more challenging problem is considered, estimating the source location of an instantaneous release using point-wise concentration observations from a mobile sensor. In this scenario, reactive or control based algorithms will be unable to track towards the source, still, they may be able to track the instantaneous puff of material downwind of the release which could also provide a useful response. The goal of this work however, is to estimate the source term of the release. This encapsulates all of the information required to produce a forecast of the spread of the material using an atmospheric transport and dispersion (ATD) model. It is also of importance in understanding the cause of the release in an emergency event. As a minimum, the details required are the release mass and the location/origin of the release source. Other important variables that can be included will depend on the scenario and the chosen ATD model including: stack height, uncertain meteorological variables, release time and the duration of the release.

The authors are with the Department of Aeronautical and Automotive Engineering, Loughborough University, Leicestershire, UK \{m.hutchinson2, c.liu5, w.chen\}alboro.ac.uk
Several source term estimation (STE) methods have been presented in the literature. Typically using a static network of sensors on the ground. Concentration observations are compared with expected observations that are obtained by running hypothesised source terms in an ATD model. The differences are compared using distance metrics or likelihood functions and then new improved hypotheses are generated [4]. Optimisation algorithms have been proposed to optimise point estimates of the source and Bayesian based approaches have been used to approximate probability distributions of the source term parameters as reviewed in [5]. Most methods have dealt with continuous releases, predominantly because it enables use of the simple Gaussian plume dispersion model. Instantaneous and non-continuous releases have been dealt with in [6] and [7], using a static network of sensors and optimisation based algorithms. The Gaussian plume dispersion model was replaced by a Gaussian puff model and new parameters were introduced into the optimisation such as the start time and the duration of the release. In this work, we shall estimate the parameters using a Bayesian based approach and a single mobile sensor instead of a static network. It is assumed the sensor is installed on an unmanned aerial vehicle (UAV) which has adequate speed to track the dispersion.

Since the source can not be tracked towards directly, the model based STE techniques must be applied to estimate the source location of the instantaneous or puff release. Several limitations and opportunities arise when applying a mobile sensor on a UAV to the problem. The main limitation will be with respect to the start time of the release, the wind speed and the time that the UAV is deployed, where the UAV may simply have to "chase" the puff downwind, until some positive readings of concentration are found. Spatialtemporal measurements of the hazard can be improved but are also limited if there is only one mobile sensor. The advantages arise from the movement of the sensor, so that measurements can be taken from more desirable locations.

Mobile sensors have been applied previously to source term estimation problems where it has been assumed that the source is continuously releasing. For example, in [8] two ground robots were used to estimate the source terms of multiple radiological releases. The robots moved sequentially by maximising a reward based on the fisher information. In [9] an expert system was used to estimate the parameters of a dispersing source, where the location and strength of the source were estimated using a genetic algorithm. Finally in [10], the parameters of a hazardous dispersing source were estimated using a Markov chain Monte Carlo algorithm, 
where the sensor platform moved by maximising a reward that was based on maximum entropy sampling principles. The only record of application of mobile sensors to estimate the parameters of an instantaneous release is found in [11], however it had been assumed that the source location was known a priori, so only the strength of the release was estimated.

In this work, STE methods using mobile sensors are extended to estimate the parameters of an instantaneous release in uncertain meteorological conditions. Spatially, the problem is extended to three dimensions in the estimation and sensor planning algorithms. Bayes' theorem is used to estimate the parameters of the source in the presence of uncertain meteorological and dispersion variables. A reward based on the Kullback-Leibler divergence is used for sensor planning. The algorithm is implemented using the sequential Monte Carlo framework. Simulations are presented to compare the performance of the system in comparison to using a static network of sensors.

The remainder of the paper is organised as follows. In Section 2, the problem is presented including information about the domain and modelling used within the algorithm and for the simulations. In Section 3, the conceptual solution is described. In Section 4, we outline the computational algorithms used to implement the conceptual solution. An illustrative run and Monte Carlo simulations with other strategies are presented in Section 5, and finally, the paper is concluded and ideas for future work are put forward in Section 6.

\section{PROBLEM DESCRIPTION AND MODELLING}

An instantaneous or puff release undergoing atmospheric transport and dispersion can be characterised by the Gaussian puff equation [7] using the source term vector $\theta=$

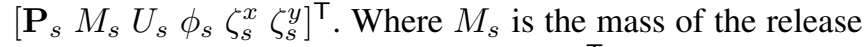
with origin position $\mathbf{P}_{s}=\left[x_{s}, y_{s}, z_{s}\right]^{\top}$. $U_{s}$ is the wind speed, $\phi_{s}$ is the wind direction, and $\left(\zeta_{s}^{x}, \zeta_{s}^{y}\right)$ are stochastic dispersion parameters adopted from [12].

A sensor equipped UAV has the position vector $\mathbf{P}_{k}=$ $\left[x_{k}, y_{k}, z_{k}, t_{k}\right]^{\top}$ (where $x_{k}, y_{k}, z_{k}$ are the Cartesian coordinates of the sensor at time $t_{k}$ ) is to make concentration observations to estimate the release parameters. The mean concentration observed by the sensor from an instantaneous source $\theta$ can be modelled using the Gaussian Puff model as:

$$
\begin{aligned}
C\left(\mathbf{P}_{k}, \theta\right) & =\frac{M}{(2 \pi)^{\frac{3}{2}} \sigma_{x} \sigma_{y} \sigma_{z}} \exp \left[-\frac{\left(x_{k}-x_{c}\right)^{2}}{2 \sigma_{x}^{2}}-\frac{\left(y_{k}-y_{c}\right)^{2}}{2 \sigma_{y}^{2}}\right] \\
& \times\left(\exp \left[-\frac{\left(z_{k}-z_{s}\right)^{2}}{2 \sigma_{z}^{2}}\right]+\exp \left[-\frac{\left(z_{k}+z_{s}\right)^{2}}{2 \sigma_{z}^{2}}\right]\right)
\end{aligned}
$$

where $x_{c}$ and $y_{c}$ are the coordinates of the centroid of the puff that is translated by the wind over time, defined as:

$$
\begin{aligned}
& x_{c}=x_{s}-U_{s} \sin \left(\phi_{s}\right)\left(t-t_{s}\right) \\
& y_{c}=y_{s}-U_{s} \cos \left(\phi_{s}\right)\left(t-t_{s}\right)
\end{aligned}
$$

and $\left(\sigma_{x}, \sigma_{y}, \sigma_{z}\right)$ are dispersion parameters defined as a function of downwind distance $\bar{x}$ using the Karlsruhe-Jülich system [13] as:

$$
\sigma_{x}=\sigma_{y}=a \bar{x}^{b} \quad \text { and } \quad \sigma_{z}=c \bar{x}^{d} .
$$

The variables $(a, b, c, d)$ are a function of stability category [14]. For example at Pascal stability category C: $a=$ $0.66, b=0.81, c=0.17$ and $d=1$. Inspired by the work in [12], where the constants in the dispersion parameter equations were replaced by stochastic parameters $\left(\zeta_{1}, \zeta_{2}\right)$ the equations for the dispersion parameters are reformulated as:

$$
\sigma_{x}=\sigma_{y}=\zeta_{1} x^{b} \quad \text { and } \quad \sigma_{z}=\zeta_{2} x^{d},
$$

where $b$ and $d$ are still selected based on the stability class.

An example run of the Gaussian puff model from Eq (1) is shown in Fig 1, where the red dot denotes the source position and the colour map represents the concentration at positions in the $\mathrm{x}, \mathrm{y}$ and $\mathrm{z}$ frames. The figure shows examples 50 seconds and 300 seconds after the release, with a $4 \mathrm{~m} / \mathrm{s}$ wind speed directed 20 degrees from the $\mathrm{x}$ axis.
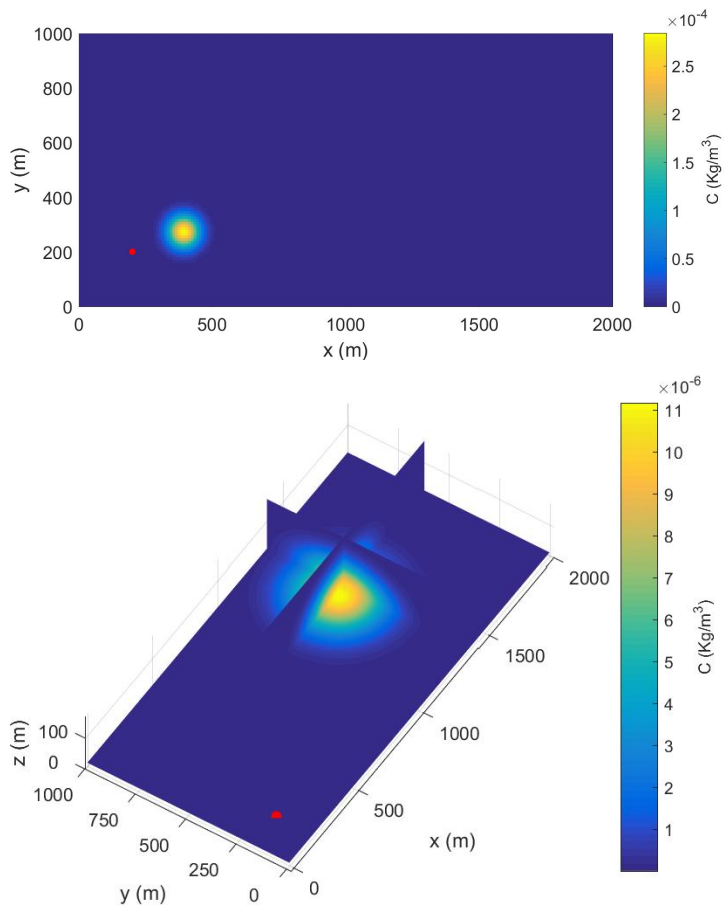

Fig. 1. Example plot of the concentration from the puff model at a) 50 seconds and b) 300 seconds after the release. The red dot indicates the origin of the source and the colour map denotes the concentration at the correspondence position, generated from a puff with parameters: $\mathbf{P}_{s}=[200,200,1]^{\top}, M_{s}=150, U_{s}=4, \phi_{s}=20$ and $[a, b, c, d]=$ $[0.14,0.95,0.53,0.73]$.

At time step $k$ the UAV will be at the position $\mathbf{P}_{k}$, the sensor observes a concentration $D$ defined as:

$$
D_{k}=D_{k}^{\text {true }}+e
$$

where $e$ refers to the error in the measurement. The observations of concentration from a sensor $D_{k}$ and from predictions with a model $C\left(\mathbf{P}_{k}, \theta\right)$ are infected with several sources of 
error that can arise from sensor noise and drift, modelling errors or errors in other dispersion variables such as the wind speed. An appropriate distance metric or likelihood function must determine the probability of the observed data given an expected reading from the model. Several distributions have been used in the past. Application of the maximum entropy principle [15] suggests that the most conservative choice of likelihood function is Gaussian. This leads to the following likelihood function between observed and modelled concentrations which encapsulates the errors from modelling and sensing:

$$
P\left(D_{k} \mid \theta\right)=\frac{1}{\sigma_{k}\left(D_{k}\right) \sqrt{2 \pi}} \exp \left[-\frac{\left(D_{k}-C\left(\mathbf{P}_{k}, \theta\right)\right)^{2}}{2\left(\sigma_{k}\left(D_{k}\right)\right)^{2}}\right],
$$

where the variance is defined as: $\sigma_{k}\left(D_{k}\right)=0.1 D_{k}$. In the next section, Bayes' theorem is introduced which will use the likelihood function to update estimates of the source parameters.

\section{CONCEPTUAL SOLUTION}

A sensor equipped UAV is released to estimate the parameters of the Gaussian puff. At each time step the parameters of the source are estimated using Bayes' theorem [5] and then the sensor chooses the next position to take a measurement by maximising an information based reward. Bayes' theorem is chosen to estimate the source parameters as it can be robust to uncertainty as the errors expected in the observations can be modelled to reflect such uncertain conditions. We have chosen an information based reward as it takes into account the effect that the future measurement may have on the estimates of the source parameters. Furthermore, it has previously been shown to be effective for continuously releasing scenarios [10].

Using Bayes' theorem, the posterior distribution of the source parameters $P\left(\theta \mid \mathbf{D}_{1: k}\right)$ is updated recursively as new sensor observations become available as:

$$
P\left(\theta \mid \mathbf{D}_{1: k}\right)=\frac{P\left(D_{k} \mid \theta\right) P\left(\theta \mid \mathbf{D}_{1: k-1}\right)}{P\left(D_{k} \mid \mathbf{D}_{1: k-1}\right)},
$$

where

$$
P\left(D_{k} \mid \mathbf{D}_{1: k-1}\right)=\int P\left(D_{k} \mid \theta\right) P\left(\theta \mid \mathbf{D}_{1: k-1}\right) \mathrm{d} \theta .
$$

At each iteration, the previous result replaces the posterior distributions of the parameters. To initialise the algorithm, prior distributions for each of the parameters in the source vector must first be selected. Where possible, these can be given informative distributions. For example, assuming that meteorological sensors are available, a normal distribution is used for the prior on the wind speed $U_{s}$ and direction $\phi_{s}$. The remaining priors are set as uniform distributions within some reasonable bounds. Bayes' theorem is implemented using a particle filter as described in the implementation section.

Once the posterior distribution at the current time step is obtained, 6he UAV must choose where to take the next measurement by maximising the expected gain in information:

$$
\mathbf{a}_{k}^{*}=\arg \max _{\mathbf{a}_{k} \in \Psi}\left\{E\left(u\left(\mathbf{a}_{k}\right)\right)\right\}
$$

where $\mathbf{a}_{k} \in \Psi$ is the set of manoeuvres that the UAV can make. In this paper, the manoeuvre set is limited to a single move in the $\mathrm{x}, \mathrm{y}$, or $\mathrm{z}$ directions with a fixed step size: $\Psi=$ $\{+x,-x,+y,-y,+z,-z\}$.

Inspired by the work on optimal experiment design, the expected utility of manoeuvre $\mathbf{a}_{k}$ is given as the product of the likelihood of an observation/measurement and its corresponding utility $u\left(\theta, \hat{D}_{k+1}, \mathbf{a}_{k}\right)[?]$ :

$$
E\left(u\left(\mathbf{a}_{k}\right)\right)=\int_{\hat{D}_{k+1}} p\left(\hat{D}_{k+1} \mid \theta, \mathbf{a}_{k}\right) u\left(\theta, \hat{D}_{k+1}, \mathbf{a}_{k}\right) d \hat{D}_{k+1},
$$

where $\hat{D}_{k+1}$ is the range of possible future measurements at the potential sampling position. The utility of the manoeuvre is defined as the Kullback-Leibler divergence between the source parameter distributions before $p(\theta)$ and after $p\left(\theta \mid \hat{D}_{k+1}, \mathbf{a}_{k}\right)$ the new measurement.

$$
\begin{aligned}
u\left(\theta, \hat{D}_{k+1}, \mathbf{a}_{k}\right)=D_{K L}\left(p\left(\theta \mid \hat{D}_{k+1}, \mathbf{a}_{k}\right) \| p(\theta)\right) \\
=\int_{\Theta} p\left(\theta \mid \hat{D}_{k+1}, \mathbf{a}_{k}\right) \ln \frac{p\left(\theta \mid \hat{D}_{k+1}, \mathbf{a}_{k}\right)}{p(\theta)} d \theta
\end{aligned}
$$

Combining (11) and (12) leads to the following expression for the reward function.

$$
\begin{array}{r}
E\left(u\left(\mathbf{a}_{k}\right)\right)=\int_{\hat{D}_{k+1}} p\left(\hat{D}_{k+1} \mid \theta, \mathbf{a}_{k}\right) \int_{\Theta} p\left(\theta \mid \hat{D}_{k+1}, \mathbf{a}_{k}\right) \\
\ln \frac{p\left(\theta \mid \hat{D}_{k+1}, \mathbf{a}_{k}\right)}{p(\theta)} d \theta d \hat{D}_{k+1}
\end{array}
$$

The complex double integral in $\mathrm{Eq}(13)$ can be approximated efficiently by importance sampling as explained in the next section.

\section{IMPLEMENTATION}

A Particle filter is used to approximate the posterior distribution of the source parameters [16]. Equation (8) is represented by a weighted random sample $\left\{\left(\theta_{k}^{(i)}, w_{k}^{(i)}\right)\right\}_{1 \leq i \leq N}$, where $\theta_{k}^{(i)}=\left[\begin{array}{ll}x_{k}^{(i)} & y_{k}^{(i)} z_{k}^{(i)} M_{k}^{(i)} U_{k}^{(i)} \phi_{k}^{(i)} \zeta_{1, k}^{(i)} \zeta_{2, k}^{(i)}\end{array}\right]^{\top}$ is a point estimate representing a potential source term and $w_{k}^{(i)}$ is the corresponding normalised weighting. Given the weighted samples, the posterior distribution can be approximated as:

$$
P\left(\theta_{k} \mid \mathbf{D}_{1: k}\right) \approx \sum_{i=1}^{N} w_{k}^{(i)} \delta\left(\theta-\theta_{k}^{(i)}\right),
$$

The unnormalised weights are updated according to:

$$
\bar{w}_{k}^{(i)}=w_{k-1}^{(i)} \cdot \frac{P\left(\theta_{k}^{(i)} \mid \theta_{k-1}^{(i)}\right) P\left(D_{k} \mid \theta_{k}^{(i)}\right)}{q\left(\theta_{k}^{(i)} \mid \theta_{k-1}^{(i)}, D_{k}\right)} .
$$

Assuming the source parameters are static (hence $\theta_{k}^{(i)}=$ $\left.\theta_{k-1}^{(i)}\right)$, Eq (15) can be simplified to:

$$
\bar{w}_{k}^{(i)}=w_{k-1}^{(i)} \cdot P\left(D_{k} \mid \theta_{k}^{(i)}\right)
$$

To avoid sample degeneracy, at each iteration the number of effective samples are approximated as $N_{\text {eff }}=$ $1 / \sum_{i=1}^{N}\left(w_{k}^{(i)}\right)^{2}$, when this falls below a threshold the particles are re-sampled. To improve the diversity of the samples, 
the re-sampled particles are moved using a metropolis Hastings Markov chain Monte Carlo move step [17].

The reward in Eq(13) must be integrated over values of the future measurement $\hat{D}_{k+1}$. This is approximated by taking $M$ samples from the current posterior distribution of the source parameters and running them in the dispersion model producing $\left\{\hat{D}_{k+1}^{(j)}\right\}_{1 \leq j \leq M}$. Where $\hat{D}_{k+1}^{(j)}$ is the result of running a source term at the considered future measurement position given by manoeuvre $\mathbf{a}_{k}$. Given these samples Eq (11) can be approximated via Monte Carlo:

$$
E\left(\hat{u}\left(\mathbf{a}_{k}\right)\right) \approx \frac{1}{M} \sum_{j=1}^{M} u\left(\theta_{k}^{(j)}, \hat{D}_{k+1}^{(j)}, \mathbf{a}_{k}\right) .
$$

The utility function is calculated by using the current weighted sample approximation of the posterior $\left\{\left(\theta_{k}^{(i)}, w_{k}^{(i)}\right)\right\}_{1 \leq i \leq N}$ and the corresponding updated sample $\left\{\left(\hat{\theta}_{k}^{(i)}, \hat{w}_{k+1}^{(i, j)}\right)\right\}_{1 \leq i \leq N}$ that represents the new posterior distribution, if the robot was to take manoeuvre $\mathbf{a}_{k}$ and observe the hypothesised measurement $\hat{D}_{k+1}^{(j)}$. Note that the sample points do not change, only the corresponding weights, i.e. $\hat{\theta}_{k}^{(i)}=\theta_{k}^{(i)}$. The expected utility is now approximated as:

$$
E\left(\hat{u}\left(\mathbf{a}_{k}\right)\right)=\frac{1}{M} \sum_{j=1}^{M} \sum_{i=1}^{N} \hat{w}_{k+1}^{(i, j)} \ln \frac{\hat{w}_{k+1}^{(i, j)}}{w_{k}^{(i)}},
$$

where $\hat{w}_{k+1}^{(i, j)} \propto w_{k}^{(i)} \cdot P\left(\hat{D}_{k+1}^{(j)} \mid \theta_{k}^{(i)}\right)$ such that $\sum_{j=1}^{M} \hat{w}_{k+1}^{(i, j)}=$ 1. The expected utility in $\mathrm{Eq}(18)$ is calculated for all values of $\mathbf{a}_{k} \in \Psi$ and the most informative manoeuvre $\mathbf{a}_{k}^{*}$ is selected as shown in Eq(10). This concludes the description of the algorithm used to estimate the source term and to guide the UAV.

\section{Simulations}

Simulations are used to assess the performance and feasibility of performing source term estimation of an instantaneous release using a single mobile sensor. The results are compared with the more common approach: using an array of varying amounts of static sensors. The simulations are designed to provide a fair comparison between the two approaches, both of which can have limitations. For example, it would not be a fair comparison if the hazardous material did not pass any or even a couple of the static sensors. Likewise, it would not be fair if the wind speed was faster than the speed of the UAV platform or if it was initialised a long time after the release or from a very poor starting position. Therefore, in this preliminary study, the simulations are constrained to a scenario where the UAV can move 2 times faster than the wind and at least half of the puff passes over the static network.

An example run is provided to demonstrate the behaviour of the algorithm during a typical source term estimation task. The scenario described will be ran multiple times to produce a Monte Carlo comparison between the mobile sensor and a static network.

\section{A. Illustrative runs}

An illustrative run of the algorithm is given in Fig 2. Simulated data was generated from the model described by $\mathrm{Eq}(1)$ and infected with Gaussian noise. An instantaneous release of $150 \mathrm{~kg}$ that occurred 50 seconds prior to the search start was simulated. The wind had a speed of $4 \mathrm{~m} / \mathrm{s}$, $20^{\circ}$ from the $\mathrm{x}$-axis. The UAV, started the search from $\mathbf{P}_{k}=[100,300,31]^{\top}$ and followed the path indicated by the red line, where red dots represent the positions where measurements were taken. The discrete time step used during the simulations was 3 seconds, during this interval, it was assumed the UAV could move $36 \mathrm{~m}$. The large number of green dots represents the random sample approximation of the location estimate of the source and the shaded contour shows the concentration of the puff at the current time. The posterior estimates of the source parameters at the end of the illustrative run are shown in the histograms in Fig 3, where the red line is the true value of the parameter. Similarly, an illustrative run is shown in Fig 4 in the same conditions, where a static network of sensors was used in place of a UAV. The static sensors were distributed on the circumference of a circle with centre $(1400,400)$ and radius 300 . The figures and histograms in Figs 3 and 5 demonstrate how the UAV can produce a more certain posterior estimate of the source.

\section{B. Results}

The results after 100 Monte Carlo simulations using the set-up illustrated in Figs 2 and 4 are summarised in Table I. Other scenarios also shown include various wind speeds $u=2,4,6$, release masses $M=75,150$ and a different amount of static sensors. The root mean squared errors (RMSEs) are shown for the mean estimates of the location $\left(x_{s}, y_{s}, z_{s}\right)$, release mass $M_{s}$ and start time $t_{s}$ of the release. The remaining variables in the source vector are not shown as they are mainly included in the vector as nuisance parameters. The benefits of using a UAV to estimate the source term in lower wind conditions are clear, however, at higher wind speeds the static network begins to outperform the UAV based system. Interestingly, the difference in the number of static sensors had less effect than expected. This may be caused by the considerable amount of data read by the sensors after the large amount of time steps. The static sensors could typically estimate the mass of the release much more accurately, this is caused by the greater amount of observations of the puff, reducing the effects of noise. Moreover, the static networks took observations simultaneously from several locations within the puff at a single instance, including observations near its centroid where the concentrations are greatest. On the other hand, the singular UAV would have less observations of the puff, which were more typically taken from its edge.

In general, more accurate estimates of the release parameters were obtained for both static and mobile sensors when the release was stronger, as shown in the Table, where the results for a release mass of $150 \mathrm{~kg}$ were more accurate than $75 \mathrm{~kg}$. For both amounts of sensors, more accurate estimates were obtained in lower wind conditions, besides those for 

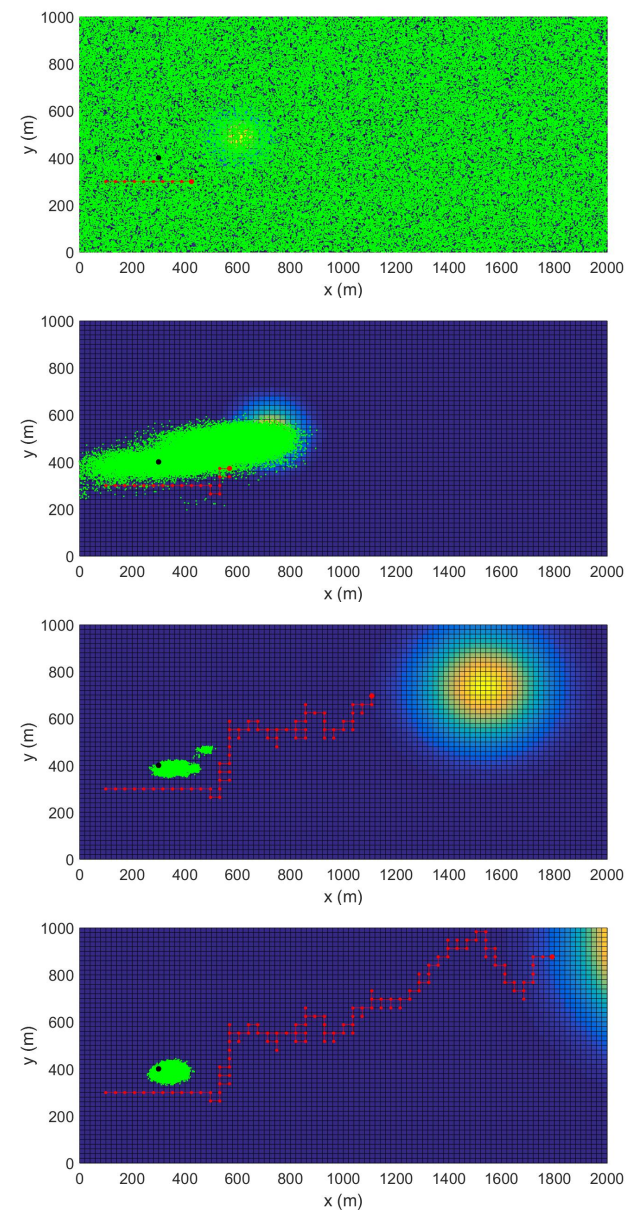

Fig. 2. Illustrative run using a single UAV at time steps a) $k=10$; b) $k=20$; c) $\mathrm{k}=90$; and d) $\mathrm{k}=160$.. The UAV starts at $(100,300)$ and follows the red lined path taking measurements at the red dots. The green dots represent the random sample approximation of the posterior distribution of the source with true position indicated by the black dot at $(300,400)$.
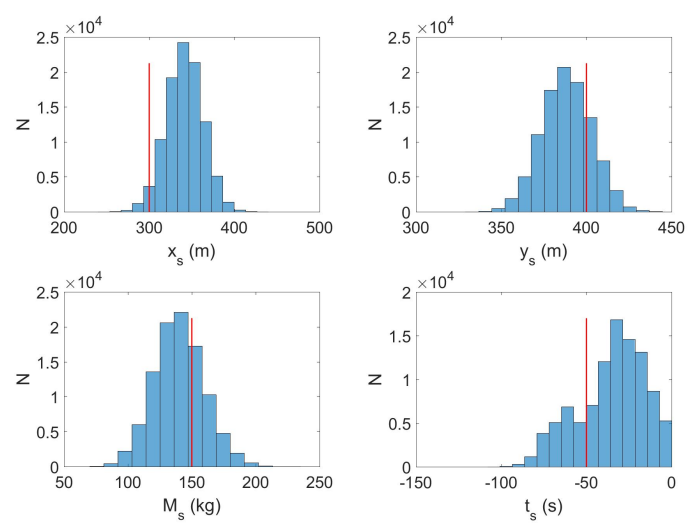

Fig. 3. Posterior density estimates at the end of the illustrative run using a single UAV for the source parameters: a-b) $x_{s}$ and $y_{s}$ coordinates; c) release mass $M_{s}$; and d) the start time $t_{s}$.

the start time of the release. This is caused by the large amount of correlation between variables estimated using the particle filter, which makes them more dependant on the prior distributions provided at the beginning of the simulations. There are several causes of correlation, for example
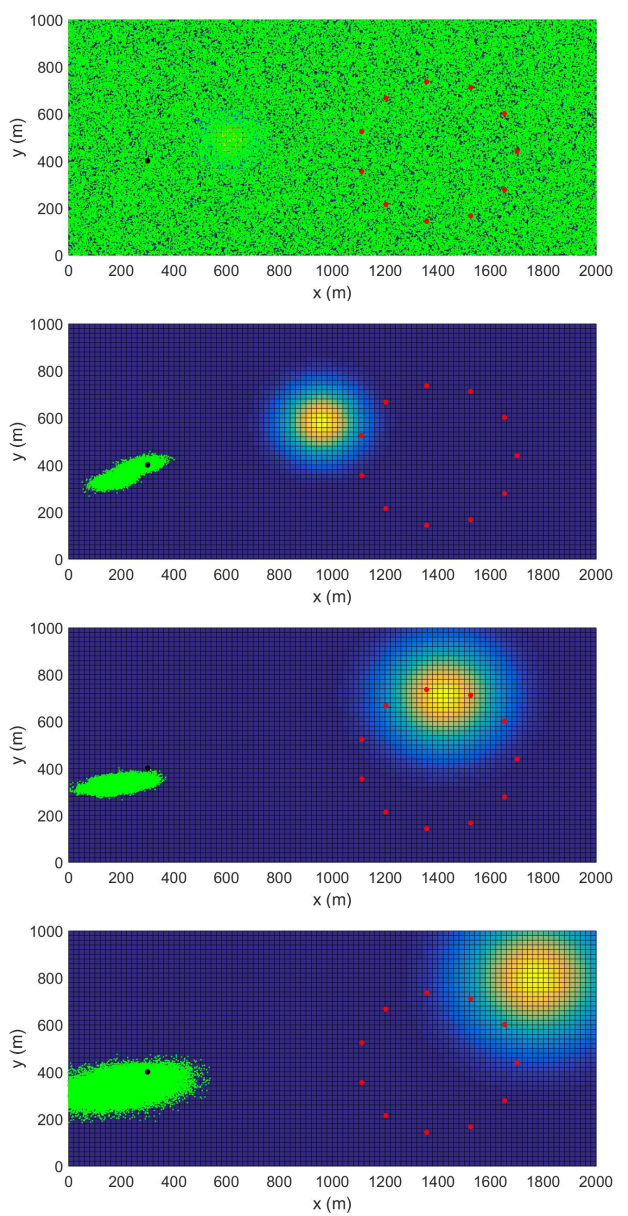

Fig. 4. Illustrative run using a circular array of 12 static sensors at time steps a) $\mathrm{k}=10$; b) $\mathrm{k}=40$; c) $\mathrm{k}=80$; and d) $\mathrm{k}=110$. Red dots denote the locations of the sensors. The green dots represent the random sample approximation of the posterior distribution of the source with true position indicated by the black dot at $(300,400)$.
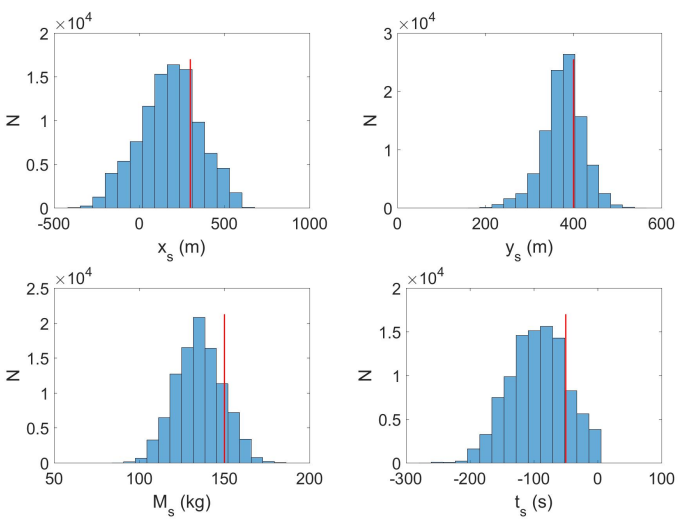

Fig. 5. Posterior density estimates at the end of the illustrative run using 12 static sensors for the source parameters: $\mathrm{a}-\mathrm{b}) x_{s}$ and $y_{s}$ coordinates; c) release mass $M_{s}$; and d) the start time $t_{s}$.

downwind location, wind speed and release time are highly correlated as is shown by Eq(2). The release time and mass of release are also correlated, where a release from long time ago produces smaller measurements, similarly to a smaller mass. 
TABLE I

Performance COMParison OVer a HUNDRed Monte CARlo Simulations

\begin{tabular}{llllllll}
\hline & True mass $M_{s}$ & 75 & 75 & 75 & 150 & 150 & 150 \\
& Wind speed $U_{s}$ & 2 & 4 & 6 & 2 & 4 & 6 \\
\hline \multirow{5}{*}{1 mobile sensor } & RMSE in $x_{s}(m)$ & 55.78 & 108.10 & 159.65 & 63.77 & 137.89 & 169.86 \\
& RMSE in $y_{s}(m)$ & 38.39 & 43.70 & 54.48 & 33.15 & 51.52 & 56.23 \\
& RMSE in $z_{s}(m)$ & 1.65 & 1.58 & 1.60 & 1.58 & 1.72 & 1.69 \\
& RMSE in $M_{s}(k g)$ & 28.14 & 27.85 & 24.58 & 76.18 & 49.18 & 27.10 \\
& RMSE in $t_{s}(s)$ & 73.44 & 29.59 & 22.17 & 60.69 & 36.11 & 28.83 \\
\hline \multirow{5}{*}{12 static sensors } & RMSE in $x_{s}(m)$ & 109.52 & 165.66 & 186.35 & 102.66 & 145.59 & 179.38 \\
& RMSE in $y_{s}(m)$ & 46.80 & 49.72 & 52.16 & 38.66 & 44.75 & 50.16 \\
& RMSE in $z_{s}(m)$ & 2.80 & 2.22 & 1.73 & 2.54 & 1.92 & 1.85 \\
& RMSE in $M_{s}(k g)$ & 10.07 & 9.73 & 5.97 & 15.18 & 8.86 & 8.29 \\
& RMSE in $t_{s}(s)$ & 53.34 & 44.11 & 33.05 & 54.81 & 42.69 & 32.53 \\
\hline \multirow{5}{*}{ s5 static sensors } & RMSE in $x_{s}(m)$ & 134.62 & 152.21 & 158.51 & 144.44 & 145.15 & 153.31 \\
& RMSE in $y_{s}(m)$ & 49.88 & 45.40 & 54.30 & 46.41 & 46.22 & 41.05 \\
& RMSE in $z_{s}(m)$ & 2.54 & 2.07 & 2.67 & 2.25 & 2.22 & 1.68 \\
& RMSE in $M_{s}(k g)$ & 20.89 & 18.38 & 16.15 & 22.76 & 18.45 & 19.83 \\
& RMSE in $t_{s}(s)$ & 89.84 & 68.63 & 35.61 & 107.73 & 51.37 & 40.11 \\
\hline
\end{tabular}

\section{CONCLUSIONS}

An approach has been proposed to estimate the source term parameters of an instantaneous release of dispersing material into the atmosphere. In simulations, the system was shown to successfully estimate the parameters of the release including its location, start time and the quantity of released material. The method was comparable in performance to that of a static network, whilst overcoming issues such as positioning of the sensors, and the costs of powering and maintenance of a large network of sensors. The UAV based system was able to estimate the parameters of the release more accurately in simulations with a wind speed less than half that of the UAV. The difficulty of estimating all the dispersion parameters of a puff due to coupling between variables was highlighted and should be addressed in future work. Further simulations will be required to test the algorithm with alternative starting conditions. These may be built from further information regarding the operational circumstances the system is expected to be deployed into.

There are still some limitations of the system, such as the speed of the UAV for which the dynamics have so far been ignored, and we assumed that it was known that the release was an instantaneous puff a priori. In the future the approach should be extended to scenarios where the duration of the release is unknown. We envisage a hybrid state or multiple model based particle filter could handle this scenario.

\section{ACKNOWLEDGEMENTS}

This work was supported by the UK Engineering and Physical Sciences Research Council (EPSRC) and the Ministry of Defence (MOD) University Defence Research Collaboration in Signal Processing under the grant number $\mathrm{EP} / \mathrm{K} 014307 / 1$.

\section{REFERENCES}

[1] Bayat, B., Crasta, N., Crespi, A., Pascoal, A.M. and Ijspeert, A., 2017. Environmental monitoring using autonomous vehicles: a survey of recent searching techniques. Current Opinion in Biotechnology, 45, pp.76-84.
[2] Vergassola, M., Villermaux, E. and Shraiman, B.I., 2007. 'Infotaxis' as a strategy for searching without gradients. Nature, 445(7126), p.406.

[3] Ishida, H., Wada, Y. and Matsukura, H., 2012. Chemical sensing in robotic applications: A review. IEEE Sensors Journal, 12(11), pp.31633173.

[4] Wang, Y., Huang, H., Huang, L. and Ristic, B., 2017. Evaluation of Bayesian source estimation methods with Prairie Grass observations and Gaussian plume model: A comparison of likelihood functions and distance measures. Atmospheric Environment, 152, pp.519-530.

[5] Hutchinson, M., Oh, H. and Chen, W.H., 2017. A review of source term estimation methods for atmospheric dispersion events using static or mobile sensors. Information Fusion, 36, pp.130-148.

[6] Annunzio, A.J., Young, G.S. and Haupt, S.E., 2012. A Multi-Entity Field Approximation to determine the source location of multiple atmospheric contaminant releases. Atmospheric environment, 62 , pp.593-604.

[7] Bieringer, P.E., Rodriguez, L.M., Vandenberghe, F., Hurst, J.G., Bieberbach, G., Sykes, I., Hannan, J.R., Zaragoza, J. and Fry, R.N., 2015. Automated source term and wind parameter estimation for atmospheric transport and dispersion applications. Atmospheric Environment, 122, pp.206-219.

[8] Ristic, B., Morelande, M. and Gunatilaka, A., 2010. Information driven search for point sources of gamma radiation. Signal Processing, 90(4), pp.1225-1239.

[9] Kuroki, Y., Young, G.S. and Haupt, S.E., 2010. UAV navigation by an expert system for contaminant mapping with a genetic algorithm. Expert Systems with Applications, 37(6), pp.4687-4697.

[10] M. Hutchinson, H. Oh, and W.H. Chen, 2017. Adaptive Bayesian sensor motion planning for hazardous source term reconstruction. IFAC World Congress, France.

[11] Madankan, R., Singla, P. and Singh, T., 2015. Parameter estimation of atmospheric release incidents using maximal information collection. In Dynamic Data-Driven Environmental Systems Science (pp. 310-321). Springer, Cham.

[12] Senocak, I., Hengartner, N.W., Short, M.B. and Daniel, W.B., 2008. Stochastic event reconstruction of atmospheric contaminant dispersion using Bayesian inference.

[13] Thykier-Nielsen, S., Deme, S. and Mikkelsen, T., 1999. Description of the atmospheric dispersion module RIMPUFF. Riso National Laboratory, PO Box, 49.

[14] Panofsky, H.A. and Dutton, J.A., 1984. Atmospheric turbulence: models and methods for engineering applications (No. 04; QC880. 4. T8, P3.). Wiley. Atmospheric Environment, 42(33), pp.7718-7727.

[15] Jaynes, E.T., 2003. Probability theory: The logic of science. Cambridge university press.

[16] Arulampalam, M.S., Maskell, S., Gordon, N. and Clapp, T., 2002. A tutorial on particle filters for online nonlinear/non-Gaussian Bayesian tracking. IEEE Transactions on signal processing, 50(2), pp.174-188.

[17] Ristic, B., Arulampalam, S. and Gordon, N., 2004. Beyond the Kalman filter. IEEE Aerospace and Electronic Systems Magazine, 19(7), pp.3738. 